\title{
Identification of microRNAs associated with invasive and aggressive phenotype in cutaneous melanoma by next-generation sequencing
}

\author{
Sankhiros Babapoor ${ }^{1}$, Rong Wu², James Kozubek', Donna Auidi ${ }^{3}$, Jane M Grant-Kels ${ }^{3}$ and Soheil S Dadras ${ }^{1,3}$
}

A comprehensive repertoire of human microRNAs (miRNAs) that could be involved in early melanoma invasion into the dermis remains unknown. To this end, we sequenced small RNAs (18-30 nucleotides) isolated from an annotated series of invasive melanomas (average invasive depth, $2.0 \mathrm{~mm}$ ), common melanocytic nevi, and matched normal skin $(n=28)$. Our previously established bioinformatics pipeline identified 765 distinct mature known miRNAs and defined a set of top 40 list that clearly segregated melanomas into thin $(0.75 \mathrm{~mm})$ and thick $(2.7 \mathrm{~mm})$ groups. Among the top, miR-21-5p, let-7b-5p, let-7a-5p, miR-424-5p, miR-423-5p, miR-21-3p, miR-199b-5p, miR-182-5p, and miR-205-5p were differentially expressed between thin and thick melanomas. In a validation cohort $(n=167)$, measured expression of miR-21-5p and miR-424-5p, not previously reported in melanoma, were significantly increased in invasive compared with in situ melanomas $(P<0.0001)$. Increased miR-21-5p levels were significantly associated with invasive depth $(P=0.038)$, tumor mitotic index $(P=0.038)$, lymphovascular invasion $(P=0.0036)$, and AJCC stage $(P=0.038)$. In contrast, let-7b levels were significantly decreased in invasive and in situ melanomas compared with common and dysplastic nevi $(P<0.0001)$. Decreased let-7b levels were significantly associated with invasive depth $(P=0.011)$, Clark's level $(P=0.013)$, ulceration $(P=0.0043)$, and AJCC stage $(P=0.011)$. These results define a distinct set of miRNAs associated with invasive and aggressive melanoma phenotype.

Laboratory Investigation (2017) 97, 636-648; doi:10.1038/labinvest.2017.5; published online 20 February 2017

Notwithstanding the distinct sets of DNA and chromosomal alterations demonstrated in melanoma, the changes in the noncoding RNA transcriptome remain poorly understood. The microRNAs (miRNAs) are endogenous, noncoding small (18-24 nucleotides) RNAs, which can regulate gene expression in animals and plants by complementary base-pairing to the mRNAs of target genes to specify mRNA cleavage or translation repression. ${ }^{1}$ Growing evidence has shown that particular miRNAs function predominately as tumor suppressors, eg, let-7 family ${ }^{2,3}$ and miR-15a and $\mathrm{miR}-16{ }^{4}$ and some as oncogenes, eg, miR-17 92 cluster. ${ }^{5,6}$

A number of studies have demonstrated an evolving role of miRNAs in various aspects of melanoma biology and clinical behavior. For example, results showed a cell-specific upregulated Dicer expression in $81 \%$ of cutaneous, $80 \%$ of acrolentiginous, and $96 \%$ of metastatic melanomas compared with carcinomas or sarcomas of the skin. ${ }^{7}$ Moreover, the expression of Dicer was significantly higher in melanomas compared with benign melanocytic nevi. Dicer, a member of the RNase III family of double-stranded RNases, is a central enzyme in a multi-component miRNA biogenesis pathway where the Drosha/DGCR8 complex and Dicer act sequentially to crop long primary and precursor miRNAs into functionally mature miRNAs. ${ }^{8}$ In patients with cutaneous melanomas, Dicer upregulation was significantly associated with an increased tumor mitotic index, Breslow's depth of invasion, nodal metastasis, and a higher American Joint Committee on Caner (AJCC) clinical stage. ${ }^{7}$ Based on microarray hybridization or real-time quantitative RT-PCR (qRT-PCR), results show specific miRNAs being deregulated in some melanoma cell lines,,${ }^{9,10-13}$ in melanoma metastases, ${ }^{14}$ and in primary melanomas. ${ }^{15-17}$ Moreover, an expression signature, consisting of 18 miRNAs identified in metastatic specimens,

${ }^{1}$ Department of Genetics and Genome Sciences, University of Connecticut Health Center, Farmington, CT, USA; ${ }^{2}$ CICATS Biostatics Center, University of Connecticut Health Center, Farmington, CT, USA and ${ }^{3}$ Department of Dermatology, University of Connecticut Health Center, Farmington, CT, USA

Correspondence: Dr SS Dadras, MD, PhD, Departments of Dermatology and Genetics and Genome Sciences, University of Connecticut Health Center, 21 South Road, Farmington, CT 06030-6231, USA.

E-mail: dadras@uchc.edu

Received 28 September 2016; revised 20 December 2016; accepted 23 December 2016 
significantly correlated with longer survival in a cohort of melanoma patients. ${ }^{14}$

Next-generation sequencing of small RNAs isolated from common melanocytic nevi, thick primary melanomas ( $>4.0 \mathrm{~mm}$ in invasive depth) and metastatic melanomas to skin and lymph nodes revealed a top 40 list of key miRNAs that correctly distinguished benign from malignant melanocytic tumors. ${ }^{18}$ Among them was miR-211 whose expression was significantly decreased in melanomas compared with common nevi in an independent, validation cohort. ${ }^{18}$ miR-211 functions as a metabolic switch ${ }^{19}$ and as a potent tumor suppressor in vitro influencing gene pathways involved in cell invasion. ${ }^{20-22}$ It is located within the noncoding sequence (intron 6) of Melastatin-1 (MLSN-1) gene, ${ }^{20}$ which is expressed at high levels in melanocytic nevi but significantly reduced in metastatic melanomas with variable expression in primary lesions. ${ }^{23}$ Loss of MLSN-1 mRNA is predictive of significantly reduced disease-free survival of melanoma patients. ${ }^{24}$ A recent study demonstrated the diagnostic utility of miR-211 in situ hybridization as an ancillary test in melanoma diagnosis, with $90 \%$ sensitivity and $86.2 \%$ specificity, independent of morphologic classification. ${ }^{25}$

The miRNAs that are involved in the invasion of melanoma cell into the dermis remain understudied in clinically annotated specimens. To study melanoma dermal invasion, we sequenced small RNAs isolated from a series of annotated melanomas (average depth of $2.0 \mathrm{~mm}$ ). Unsupervised clustering clearly segregated melanomas into thin $v$ s thick groups by defining a set of top 40 miRNAs, whose measured expression levels were validated in an independent cohort. Finally, the expression of specific miRNAs significantly correlated with melanoma prognostic parameters and AJCC staging, including Breslow's depth of invasion.

\section{MATERIALS AND METHODS}

\section{Study Population and Clinical Samples}

For the discovery cohort by next-generation sequencing (NGS), the specimens consisted of biopsies or excisions of matched normal skin (NS, $n=2$ ), common melanocytic nevi $(\mathrm{CN}, n=6)$, invasive primary cutaneous melanoma (PCM, $n=19$ ), and recurrent cutaneous melanoma (MMM1, $n=1$ ) of patients undergoing curative treatment at the University of Connecticut (UConn) Health Center from 2010 to 2011 (Table 1). For the validation cohort by qRT-PCR, the specimens consisted of some matched NS $(n=12), \mathrm{CN}$ $(n=13)$, DN $(n=17)$, MIS $(n=45)$, and PCM $(n=80)$ undergoing curative treatment at the UConn Dermatology department from 2009 to 2012 (Table 2). For all cohorts, we collected detailed clinicopathologic data on melanomas such as histologic subtype, depth of invasion, ulceration, mitotic index, anatomic level of invasion, tumor-infiltrating lymphocytes, and regression as described previously. ${ }^{26}$ All the specimens were prepared from deparaffinized FFPE specimens. The sequenced specimens were shave biopsies, which were histologically selected to consist $>80 \%$ of tumor cells in the tissue section. At least two board-certified dermatopathologists confirmed all rendered diagnoses and the presence of tumor before RNA extraction. NS was defined as histologically unremarkable skin, $2 \mathrm{~cm}$ away from melanoma in excision specimens. The institutional review board of the University of Connecticut Health Center approved this protocol.

\section{Next-Generation Sequencing}

Total RNA was purified from xylene-extracted FFPE tissue sections with TRIzol (Invitrogen, Carlsbad, CA, USA) and multiplex sequencing was performed as previously described. ${ }^{18}$ To decrease the chance of sequencing unwanted ribosomal RNAs, 'poison primers' were added to the ligated products. ${ }^{27}$ Briefly, after reverse transcription, a cDNA library of mixed barcodes was generated for Illumina genome analyzer (IGA) II sequencing. The doubly ligated, purified RNA was reverse-transcribed using $150 \mathrm{U}$ of Superscript II (Invitrogen) and RT primer, (5'-ATTGATGGTGCCTACAG-3'). The resultant CDNA pool was amplified by PCR with the following primers: (forward) 5'-GATACGGCGACCACCGA GATCTACACTCTTTCCCTACACGACGCTCTTCCGATCT-3'; and (reverse) 5'-CAAGCAGAAGACGGCATACGAGCTCTT CCGATCTATTGATGGTGCCTACAG-3'. The $\sim 125$ bp PCR products were purified on a $4 \%$ agarose gel followed by gel extraction using a QiaQuick column (Qiagen, Valencia, CA, USA). In the Cluster Station, repeated rounds of polymerase amplification and subsequent denaturation generate clusters of unique sequences on the surface of the flow cell that are then transferred to the Genome Analyzer for sequencing by synthesis. Four fluorescently labeled, reversible terminating nucleotides are successively incorporated, then imaged with a high-resolution laser using TIRF (total internal reflection fluorescence) optics to eliminate background and reduce the signal-to-noise ratio.

\section{RNA Quantification and Size}

The yield and quality (260/280 O.D. ratios) of RNA were measured by a spectrophotometer (Nanodrop 2000, Thermoscientific, Wilmington, DE, USA). Small RNA size was measured using Agilent Small RNA Kit with an Agilent 2100 Bioanalyzer (Agilent Technologies, Waldbronn, Germany). The calculated range of captured small RNAs for our library preparation was $144-150 \mathrm{bp}$; only libraries passing the size criterion were sequenced.

\section{miRDeep 2.0 Analysis}

RNA sequencing data in FASTQ form was input into the microRNA-characterization software miRDeep 2.0 on a Linux platform as previously described. ${ }^{18}$ Briefly, the software used Bowtie to map the reads to the UCSC reference genome GRCh37 (browser hg19), allowing for only one mismatch and compiling stretches of hairpin sequences up to $140 \mathrm{nt}$ as microRNA precursors. The precursors are then folded into two-dimensional structures using RNAfold from Vienna RNA 
Table 1 Clinicopathologic characteristics for discovery cohort

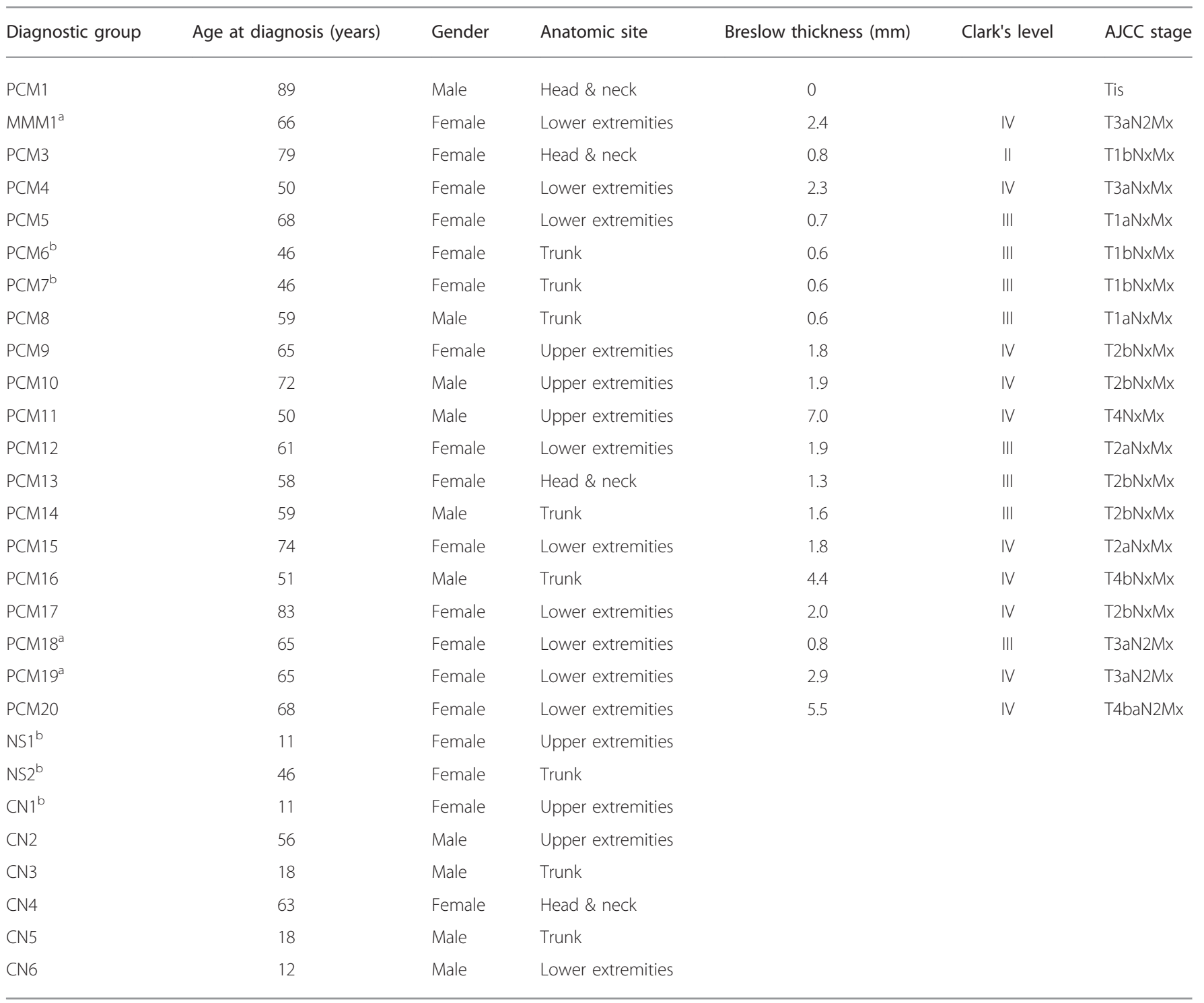

asame patient.

${ }^{\mathrm{b}}$ Matched specimens.

Table 2 Clinical characteristics for validation cohort

\begin{tabular}{|c|c|c|c|c|c|c|c|c|c|}
\hline \multirow[t]{2}{*}{ Diagnostic groups } & \multicolumn{2}{|c|}{$\begin{array}{l}\text { Age at diagnosis } \\
\text { (years) }\end{array}$} & \multicolumn{2}{|c|}{ Gender } & \multicolumn{4}{|c|}{ Anatomic sites } & \multirow[t]{2}{*}{ Total } \\
\hline & $<60$ & $\geq 60$ & Female & Male & Head \& neck & Upper extremities & Trunk & Lower extremities & \\
\hline Normal skin & 8 & 4 & 7 & 5 & 3 & 2 & 7 & 0 & 12 \\
\hline Common nevus & 10 & 3 & 6 & 7 & 0 & 1 & 11 & 1 & 13 \\
\hline Melanoma in situ & 12 & 33 & 17 & 28 & 21 & 4 & 13 & 7 & 45 \\
\hline Primary cutaneous melanoma & 37 & 43 & 31 & 49 & 14 & 28 & 29 & 9 & 80 \\
\hline Total & 82 & 85 & 72 & 95 & 39 & 36 & 75 & 17 & 167 \\
\hline
\end{tabular}


Package 1.8.5. miRDeep 2.0 evaluated structure and identified miRNAs based on: minimal free energy, length of the $3^{\prime}$ overhang, existing homologs, distribution of passenger (star), guide (mature), loop sequences, and frequency of the predominant read within the sequencing data. miRDeep 2.0 incorporates a file of known microRNAs from a curated library at miRBase and bundles corresponding isomirs together. To determine the fold difference for a specific miRNA distinguishing thin from thick invasive melanoma, we normalized the miRNA sequence count by calculating its percentage per the total sequence count for a given sample. Then normalized counts for thick melanomas were averaged and divided by the average normalized counts for thick melanomas.

\section{Nearest Shrunken Centroids}

To classify diagnostic groups using miRNAs as classifiers, we applied nearest shrunken centroids (NSC) statistical method ${ }^{28}$ as previously described. ${ }^{18}$ Briefly, miRNA sequence counts determined by miRDeep 2.0 were listed by sample and represented in a tab-delimited file as input for an R script (Prediction Analysis for Microarrays or the 'PAMR' module). This statistical module analyzed the data based on significance of miRNA expression in any single group. The sequence counts for known miRNAs were normalized using the root-mean-square or the 'scale' function in R 2.14.1. The results clustered diagnostic categories and assigned a $Z$-score statistic that was designed precisely for biomarker detection. The statistics involved the standard error for a particular miRNA added to the standard error for all miRNAs, a 'positive constant,' that stabilized the statistics and ensured that miRNAs are not highly ranked simply due to low counts (and thus their low standard error). Instead of setting $\alpha$-threshold $(P=0.05)$ a priori, we used machine learning to establish 'soft-threshold' based on a posteriori information learned through the data to best classify specimens based on the smallest number of miRNAs.

The machine-learning method selected parts of the data assigning it to a random 'test' bin. Random data were fit to expression values in one of our actual clinically defined diagnostic groups or 'training sets'. In general, misclassification decreased as more miRNAs were added to the classification process, but an optimal, minimal set of miRNAs was identified to best describe the diagnostic categories. Based on what was learned through the data, we set an absolute value threshold of 1.85 to our $Z$-scores. All scores below this absolute value were rounded to 0 and discounted; thereby, we eliminated noisiness and displayed only the most pertinent miRNAs classifying diagnostic groups. A false discovery rate (FDR) was determined by randomly permuting class assignment 100 times, thereby controlling for multiple hypothesis testing. Clustering for all samples was carried out through use of $2 \times(1-c c)$ where cc equals a correlation between the cubed root of a value, and the cubed root of a second value or the average of cube-rooted values clustered in a branch by that time point.

\section{Determining RNA Biotypes}

Sequences from UCSC annotation files wgRNA.txt (snoRNA) and tRNAs.txt (tRNA) and Biomart ${ }^{18}$ annotation files on snoRNA, tRNAs, Mt-tRNA, rRNA, snRNA, scRNA, miRNA, and lincRNA were compiled into bowtie indexes as previously described. ${ }^{18}$

\section{TaqMan miRNA Assay}

The expression profile of mature miRNAs for miR-21-5p, let-7b-5p, miR-424-5p, and miR-423-5p was measured in NS, $\mathrm{CN}, \mathrm{DN}, \mathrm{MIS}$, and PCM specimens using stem-loop primers for reverse transcription followed by qRT-PCR (TaqMan MicroRNA Assays; Applied Biosystems, Foster City, CA, USA) in a 7500 fast Sequence Detection System (Applied Biosystems). Cycle threshold (Ct) values for each miRNA were normalized $v s$ small RNA RNU6 $(\Delta \mathrm{Ct})$ and represented as $\mathrm{RQ}=2^{-\Delta \mathrm{Ct}}$. The expression levels of miRNAs were normalized against U6 noncoding small nuclear RNA. If the U6 were not amplified in a sample, the sample would get flagged and would be excluded from the study. For each sample, $5 \mathrm{ng}$ of total RNA were used for reverse transcription and $1.33 \mu \mathrm{l}$ of $15 \mu \mathrm{l}$ reverse transcription product was used for each reaction. All the experiments were carried out in triplicates with appropriate negative control.

\section{Statistical Methods}

The qRT-PCR data were log-transformed, plotted, and analyzed using statistical analysis software SAS version 9.4. Given that the data distribution was right-skewed, logarithmically transformed data were used for parallel boxplots and one-way analysis of variance (ANOVA), to compare miRNA abundance among clinic groups. When the overall test of no group differences from the ANOVA was statistically significant $(\alpha=0.05)$, post hoc pairwise comparisons with Tukey's adjustment were performed to identify group pairs that differed significantly in miRNA abundance (family level of significance $\alpha=0.05$ ). Alternatively, without logarithmic transformation, Kruskal-Wallis test was performed for overall comparisons among clinic groups $(\alpha=0.05)$. Bonferroni procedure, based on the ranks of the observations, was then used for multiple pairwise comparisons (family level of significance $\alpha=0.10$ ). These methods gave very consistent results. To assess the relationship between expression of miRNA and clinicopathologic parameters in patients with PCM, summary tables of means, standard deviation, medians, and IQR (inter-quantile range) were produced and statistical significance was tested using Wilcoxon-Mann-Whitney test for two-group comparison or Kruskal-Wallis test for comparison among $\geq 3$ groups. A two-sided alpha level of 0.05 was used. 


\section{RESULTS}

\section{Sequencing and Annotation of miRNAs}

We used two sets of independent cohorts: one for miRNA discovery by NGS and, the other for validation by qRT-PCR. For discovery, we size-selected (18-30 nt), captured, amplified, and sequenced 28 small RNA libraries. Clinical follow-up and melanoma staging were documented according to the American Joint Committee on Cancer (AJCC; Table 1). The average invasive depth for all 19 invasive PCM was $2.0 \mathrm{~mm}$. For control, libraries were prepared from some normal skin (NS) and common nevi (CN). From all 28 libraries, we obtained a total of 48932850 small RNA sequences (>17 nt), mapping to the human genome (hg19). We subjected libraries to a series of alignment searches to obtain the frequency of noncoding small RNA classes using specific databases for miRNA, mitochondrial (Mt)ribosomal RNA, transfer RNA, ribosomal RNA, small nucleolar RNA, small nuclear RNA, small cytoplasmic RNA, miscellaneous RNA (processed, uncategorized transcript), and unidentified RNA. For example, the breakdown analysis is shown for PCM1, PCM3, PCM4, and PCM5 libraries (Supplementary Figure S1), where an average 79\% (range: 68 to $80 \%$ ) of the small RNAs were identified and an average $21 \%$ (range: 8 to $31 \%$ ) were unidentified. This indicated that great majority of small RNA classes could be accurately annotated. The great majority (an average of $75 \%$, ranging 65 to $87 \%$ ) of the small RNA subclass was indeed miRNAs, representing 765 distinct mature known miRNAs. The unidentified group contained some novel sequences and some that could not be matched and were discarded from further analysis.

\section{Hierarchical Clustering of the Specimens}

To address the quality and stability of small RNAs, we applied strict size criterion using bioanalyzer results. To ensure that the library contained small RNA inserts, only those with single sharp peak (144-150 bp) were sequenced; the libraries measuring $<130 \mathrm{bp}$, failing the size criterion, were excluded and not sequenced as previously described. ${ }^{18}$ Using a series of tools, we processed the raw data showing known miRNAs, predicted novel miRNAs, and small RNA classes. The two main tools were miRDeep 2.0 (ref. 29) for assigning miRNA identity; and $\mathrm{NSC}^{28}$ for statistical analysis. miRDeep 2.0 assigned raw sequences to known miRNAs from miRBase, known miRNAs recognized by miRBase not detected by miRDeep 2.0, and predicted novel miRNAs, providing (i) lists and counts of miRNAs mapping to the mature, the passenger, and the loop sequences; (ii) possible secondary structures with predicted energy stability; and (iii) mapped positions and read counts showing isomiRs. The NSC method classified specimens by computing an average miRNA expression vector for each class. These averages were then shrunken towards the overall miRNA expression mean (centroid) across the classes to avoid overfitting the rank-ordered classifiers that were made up of only a subset of the miRNAs.
Such centroid analysis of miRNA expression identified unique miRNAs up- or downregulated in thin $v s$ thick invasive melanomas (Supplementary Figure S2).

We subjected sequence counts from all libraries to the NSC method and cross-validation (CV), which is a well-established method that repeatedly split samples into training and test sets selecting the optimal number of miRNAs as classifiers in human cancers. ${ }^{30}$ To assess the accuracy of $\mathrm{CV}$, we estimated the FDR based on the number of classifiers (not shown). Choosing the top 40 miRNAs resulted in $<15 \%$ FDR providing a rank order for miRNAs differentially expressed in all the specimens (not shown). An unsupervised clustering segregated PCMs according to the depth of Breslow's invasion: (1) PCM3, PCM5, PCM7, and PCM8 (average depth $=0.75 \mathrm{~mm}$ ) and (2) PCM17, PCM13, PCM14, PCM9, and PCM9 (average depth $=2.7 \mathrm{~mm}$, Figure 1). PCM11 $(7.0 \mathrm{~mm})$ and PCM12 $(1.9 \mathrm{~mm})$ were closer in distance to the latter group with averaged depth of $1.7 \mathrm{~mm}$ than the former. For the most part, cases of PCM clustered together separating them from those of NS and $\mathrm{CN}$, except for CN2 and PCM15. Interestingly, two samples from the same patient were clustered together, eg, PCM6 with NS2 and PCM18 with MMM1 (a recurrent cutaneous melanoma).

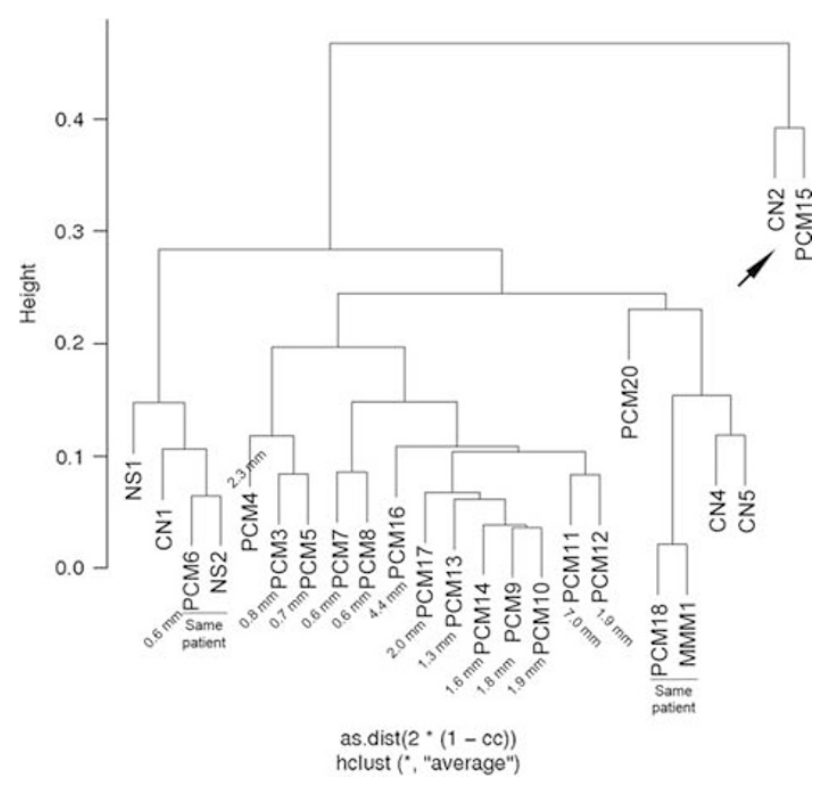

Figure 1 Hierarchical clustering of the specimens, based on sequenced miRNAs, using complete linkage analysis and correlation-based distance. Unsupervised clustering segregated invasive primary cutaneous melanomas (PCM) according to the depth of Breslow's invasion ( $\mathrm{mm}$ ). Cases PCM3, PCM5, PCM7, and PCM8 are clustered together with an average depth of $0.75 \mathrm{~mm}$, and segregated from another cluster, which is composed of PCM17, PCM13, PCM14, PCM9, and PCM9 with an average depth of $2.7 \mathrm{~mm}$. PCM11 $(7.0 \mathrm{~mm})$ and PCM12 $(1.9 \mathrm{~mm})$ were closer in distance to the latter group with an average depth of $1.7 \mathrm{~mm}$ than the former. Most cases of common nevus (CN) were clustered together from those of PCM, except for CN2 and PCM15 (arrow). MMM1 indicates local cutaneous recurrence in the same patient as PCM18. Normal skin (NS) was used as background miRNA profile. 


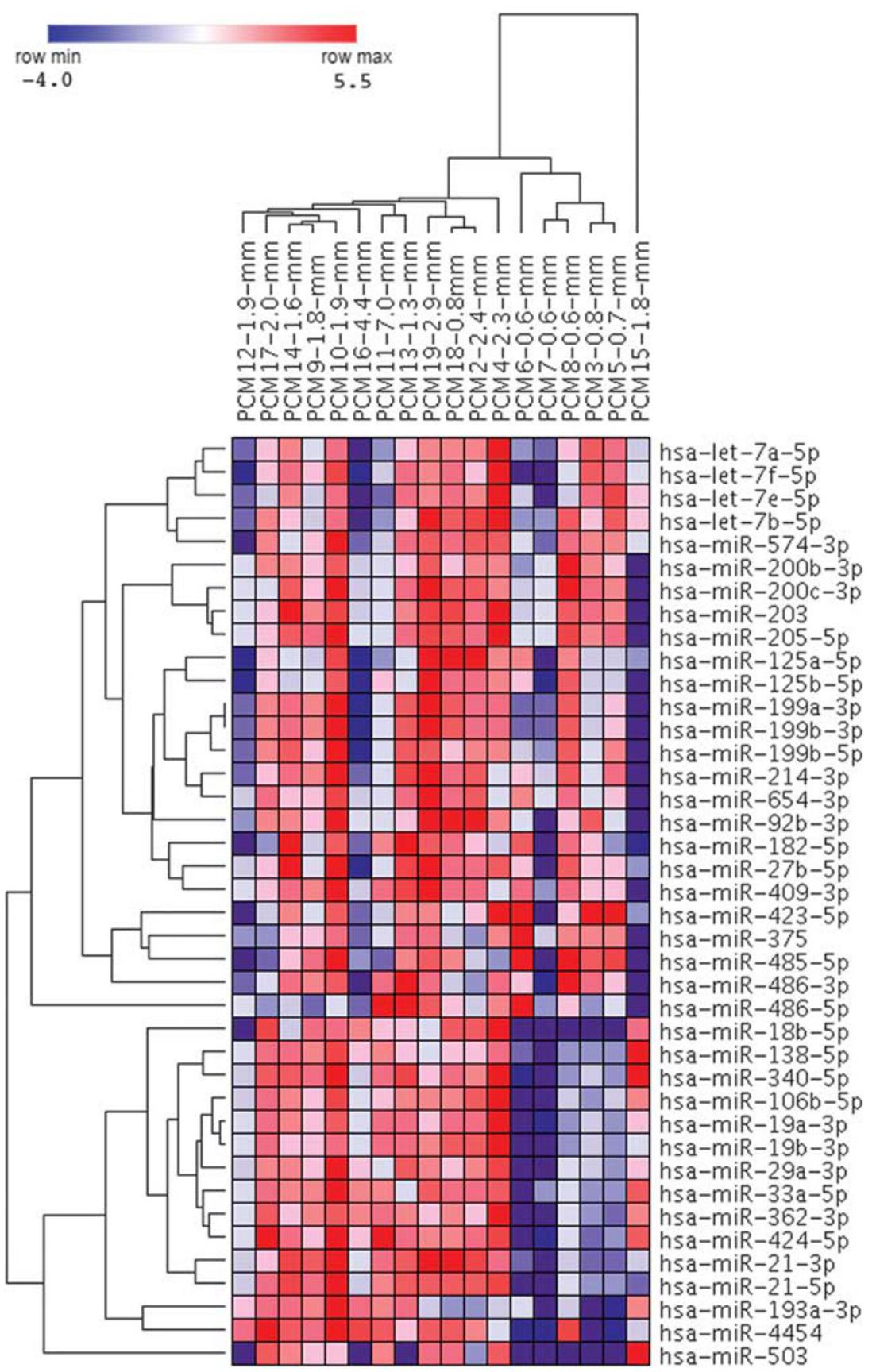

Figure 2 Heat map-clustering analysis on miRNAs in invasive melanomas. Clustering analysis of the top 40 miRNAs identified by sequencing segregated primary cutaneous melanomas (PCM) according to invasive depth using GenePattern module HierarchicalClustering. The thin PCMs with average thickness of $0.75 \mathrm{~mm}$ are segregated to the right and the thick PCMs with average thickness of $2.7 \mathrm{~mm}$ to the left. The sequence counts for PCMs were log-transformed before clustering. The rows and columns were clustered using Pearson correlation and the method was pairwise average-linkage.

Another clustering analysis using GenePattern module HierarchicalClustering ${ }^{31}$ on the top 40 miRNAs segregated all PCM into thin $(0.75 \mathrm{~mm})$ and thick $(2.7 \mathrm{~mm})$ groups (Figure 2). A specific set of miRNA expression profile (top 40 ), discovered by sequencing, could separate thin from thick invasive melanomas, which can be typically associated with a more aggressive biological potential.

\section{Verification of miRNA Expression by qRT-PCR}

To validate the miRNA NGS results in discovery cohort, we measured the expression levels of miR-21-5p, let-7b-5p, miR-424-5p, and miR-423-5p using qRT-PCR in an additional, independent cohort $(n=167$, Table 2$)$. The qRT-PCR results were expressed as RQ and shown as log values in boxplots, comparing the expression levels of the four miRNAs 
between NS, CN, DN, MIS, and PCM (Figure 3). A pairwise statistical comparison by nonparametric methods (Table 3) and Tukey (Table 4) showed a significant increase in the expression of miR-21-5p in invasive compared with in situ melanomas; and in invasive melanoma compared with dysplastic nevus (Table 3). In contrast, the expression of
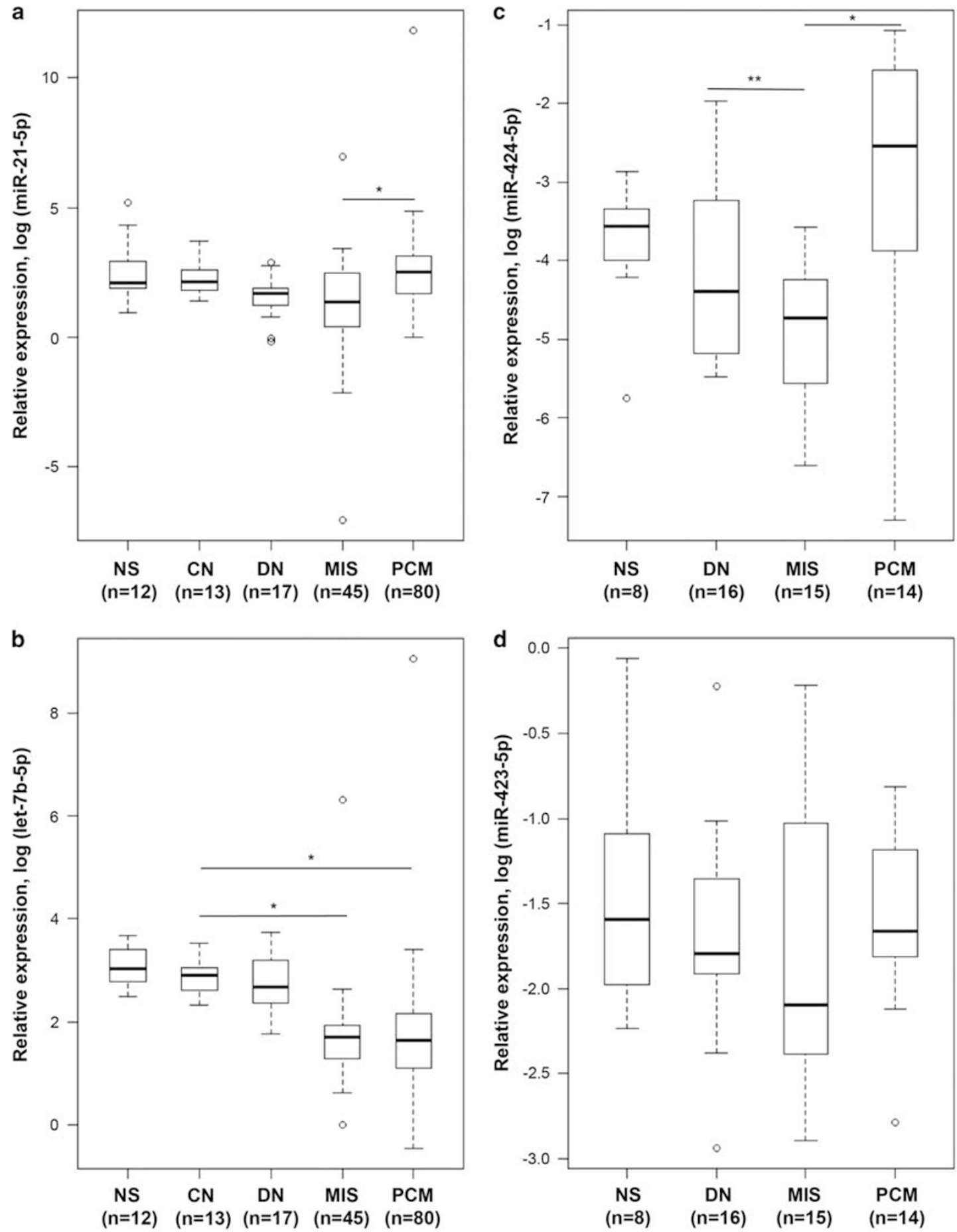

Figure 3 Verification of miRNA expression in validation cohort by qRT-PCR. Relative expression levels of miR-21-5p (a), let-7b-5p (b), miR-424-5p (c), and miR-423-5p (d), identified by sequencing, were compared between independent patient specimens of normal skin (NS), common nevus (CN), dysplastic nevus (DN), melanoma in situ (MIS), and invasive primary cutaneous melanoma (PCM). The qRT-PCR results were expressed as RQ, log-transformed and shown as log values in boxplots: box starts from first quartile and ends at third quartile; line represents mean; the ends of whiskers represent the minimum and maximum of the data that are not outliers; and circles are outliers, extreme outliers were excluded. ${ }^{*} P<0.0001 ;{ }^{* *} P=0.0088$ (see Tables 3 and 4). 
Table 3 Pairwise statistical comparisons of miRNA levels to diagnostic groups by nonparametric analysis

\begin{tabular}{|c|c|c|c|c|c|c|}
\hline miRNA & $\begin{array}{l}\text { Normal } \\
\text { skin }\end{array}$ & $\begin{array}{c}\text { Common } \\
\text { nevus }\end{array}$ & $\begin{array}{c}\text { Dysplastic } \\
\text { nevus }\end{array}$ & $\begin{array}{c}\text { Melanoma } \\
\text { in situ }\end{array}$ & $\begin{array}{c}\text { Primary } \\
\text { cutaneous } \\
\text { melanoma }\end{array}$ & $\begin{array}{l}\text { P Values* } \\
\text { (Bonferroni } \\
\text { Adjusted ) }\end{array}$ \\
\hline \multirow[t]{2}{*}{$\begin{array}{l}\text { miR-21- } \\
5 p\end{array}$} & & & & & $*$ & $<.00001$ \\
\hline & & & & & * & 0.0201 \\
\hline \multirow[t]{6}{*}{ let-7b-5p } & * & & & & & $<0.0001$ \\
\hline & * & & & & & $<0.0001$ \\
\hline & & * & & & & $<0.0001$ \\
\hline & & * & & & & $<0.0001$ \\
\hline & & & * & & & $<0.0001$ \\
\hline & & & * & & & $<0.0001$ \\
\hline \multirow[t]{3}{*}{$\begin{array}{l}\text { miR-424- } \\
5 p\end{array}$} & & & & & * & 0.0088 \\
\hline & & & & & * & $<0.0001$ \\
\hline & * & & & & & 0.0724 \\
\hline
\end{tabular}

*The Bonferroni adjustment was performed for all the pairwise comparisons of observation ranks. Shown here are only pairwise comparisons that show statistical significance (ie, $P \leq 0.05$ ). The pair with significant difference is shown with same color. ${ }^{*}$ The group with higher miRNA levels.

Table 4 Pairwise comparisons by method 1 (Tukey procedure)

\begin{tabular}{|c|c|c|c|c|c|c|}
\hline miRNA & $\begin{array}{l}\text { Normal } \\
\text { skin }\end{array}$ & $\begin{array}{c}\text { Common } \\
\text { nevus }\end{array}$ & $\begin{array}{c}\text { Dysplastic } \\
\text { nevus }\end{array}$ & $\begin{array}{l}\text { Melanoma } \\
\text { in situ }\end{array}$ & $\begin{array}{c}\text { Primary } \\
\text { cutaneous } \\
\text { melanoma }\end{array}$ & $\begin{array}{l}\text { P Values* } \\
\text { (Tukey } \\
\text { Adjusted ) }\end{array}$ \\
\hline $\begin{array}{c}\text { miR-21- } \\
5 p\end{array}$ & & & & & * & 0.0003 \\
\hline \multirow[t]{6}{*}{ let-7b-5p } & * & & & & & 0.0004 \\
\hline & * & & & & & $<0.0001$ \\
\hline & & * & & & & 0.0035 \\
\hline & & * & & & & 0.0009 \\
\hline & & & * & & & 0.0025 \\
\hline & & & * & & & 0.0005 \\
\hline \multirow[t]{2}{*}{$\begin{array}{c}\text { miR-424- } \\
5 p \\
\end{array}$} & & & & & * & 0.0144 \\
\hline & & & & & * & 0.0001 \\
\hline
\end{tabular}

*The Tukey adjustment was performed for all the pairwise comparisons of log-transformed values. Shown here are only pairwise comparisons that show statistical significance (ie, $P \leq 0.05$ ). For miR-423, there is no significant difference between four diagnostic groups.

let-7b-5p was significantly decreased in invasive and in situ melanomas compared with common and dysplastic nevi (Tables 3 and 4). Similar to miR-21-5p, the expression of miR-424-5p was significantly increased in invasive compared with in situ melanomas and in dysplastic nevi (Tables 3 and 4). For miR-423-5p, there was no significant difference between five diagnostic groups. We sought whether this differential expression could reliably segregate benign from 
malignant by receiver operating characteristic (ROC) curves. The results were as follows for miR-21: (1) invasive compared with in situ melanomas $(\mathrm{AUC}=0.72)$ and (2) invasive melanomas compared with dysplastic nevi $(\mathrm{AUC}=0.73)$. After removing extreme outliers, the ROC curves for let-7b were: (1) invasive melanomas compared with dysplastic nevi $(\mathrm{AUC}=0.89)$ and in situ melanomas compared with dysplastic nevi $(A U C=0.98)$. Overall, the qRT-PCR results verified the direction of change in the expression of three of four selected miRNAs discovered by sequencing.

\section{Significant Correlation between miRNAs and Invasive and Aggressive Phenotype}

We correlated miRNA expression with clinical and melanoma AJCC prognostic parameters in all 80 invasive melanomas (Table 5). The expression of let-7b-5p was only associated with age (higher in patients older than 60 years of age), but not gender or anatomic site. The expression of let-7b-5p correlated with Breslow's depth of invasion (higher in lesions thicker than $1.0 \mathrm{~mm}$ ), Clark's anatomic level (highest in level IV), ulceration (higher in non-ulcerated), and AJCC pathologic stage (higher in stage T2b and beyond). In contrast, the expression of miR-21-5p correlated with Breslow's depth of invasion (higher in lesions thicker than $1.0 \mathrm{~mm}$ ), mitotic index (higher in lesions with more than one mitosis), lymphovascular invasion, and AJCC pathologic stage (higher in stage T2b and beyond; Table 6). The statistical analyses established a significant correlation between let-7b-5p and

Table 5 Summarized comparison between let-7b-5p and miR-21-5p expression and their relationship to prognostic parameters in patients with invasive primary cutaneous melanoma

\begin{tabular}{lll}
\hline & \multicolumn{2}{c}{$\begin{array}{c}\text { P-values (nonparametric } \\
\text { methods) }\end{array}$} \\
\cline { 2 - 3 } & let-7b-5p & miR-21-5p \\
\hline Gender & 0.068 & 0.72 \\
Age & $\mathbf{0 . 0 1 5}$ & 0.21 \\
Anatomic site & 0.53 & 0.58 \\
Breslow's thickness (mm) & $\mathbf{0 . 0 1 1}$ & $\mathbf{0 . 0 3 8}$ \\
Clark's level & $\mathbf{0 . 0 1 3 3}$ & 0.073 \\
Mitoses (per mm ${ }^{2}$ ) & 0.22 & $\mathbf{0 . 0 3 8}$ \\
Tumor-infiltrating lymphocytes & 0.11 & 0.69 \\
Ulceration & $\mathbf{0 . 0 0 4 3}$ & 0.26 \\
Regression & 0.43 & 0.46 \\
Lymphovascular invasion & 0.25 & $\mathbf{0 . 0 0 3 6}$ \\
AJCC pathologic stage & $\mathbf{0 . 0 1 1}$ & $\mathbf{0 . 0 3 8}$ \\
\hline
\end{tabular}

Wilcoxon-Mann-Whitney test was used for two-group comparison. Bold type signifies statistically significant $P$ values.
miR-21-5p expression and a more aggressive melanoma prognostic features.

Next we compared the expression of miR-21-5p in multiple melanocytic tumors to their histopathology from the same individual. A 50-year-old man, without family history of melanoma or dysplastic nevi, presented to our Dermatology clinic with history of four recently diagnosed melanomas, one on the right upper arm $(0.4 \mathrm{~mm}$, level II) and three on the abdomen: (1) midabdomen $(0.7 \mathrm{~mm}, 4$ mitoses $/ \mathrm{mm}^{2}$, non-ulcerated, regressing), (2) right abdomen $\left(0.3 \mathrm{~mm}, 0\right.$ mitoses $/ \mathrm{mm}^{2}$, non-ulcerated, regressing), and (3) midabdomen 2 (melanoma in situ with features of severely dysplastic nevus). In addition, he had three prior biopsies on the abdomen showing severely dysplastic nevi. On exam, he had a pigmented patch on the right anterior, lateral and posterior trunk with darker background skin speckled with innumerable, clustered dark to light brown macules ranging from 3 to $11 \mathrm{~mm}$ in diameter. The histopathology of additional biopsies revealed two additional invasive melanomas (1.6 and $0.3 \mathrm{~mm}$ in invasive depth) and two more dysplastic nevi (Figure 4a). The expression of miR-21-5p was compared between his normal skin, dysplastic nevi and previous and current invasive melanomas. His normal skin specimens showed the lowest expression of miR-21-5p, whereas a progressively increased expression was detected in melanomas with increasing dermal depth of invasion (Figure $4 \mathrm{~b}$ ). The histopathology of one of his dysplastic nevi showed the expected architectural and cytological atypia with a marked band-like inflammation in the papillary dermis (Figure 4c). The histopathologies of two invasive melanomas are illustrated (Figure $4 \mathrm{~d}-\mathrm{g}$ ).

\section{DISCUSSION}

Previously, we have established a next-generation sequencing strategy and bioinformatics pipeline that led to the discovery of known and putative novel candidate miRNAs in a series of melanocytic nevi, thick primary $(>4.0 \mathrm{~mm})$ and metastatic melanomas. ${ }^{18}$ Using the whole section, without tumor microdissection, we showed that characterizing the miRNA transcriptome in 19 specimens classified the melanocytic nevi from malignant melanomas. These results were independently verified by qRT-PCR in additional 101 specimens. ${ }^{18}$ Even though we used whole sections, the sequenced specimens were shave biopsies, which were histologically selected to consist $>80 \%$ of tumor cells in the tissue section. Comparable to our methodology, micro-dissection is currently not used in other published papers where whole-cell RNA was extracted from FFPE prostate cancer sections ${ }^{32}$ or whole blood was used to detect miR-345 in patients with metastatic colorectal cancer who did not respond to cetuximab and irinotecan treatments. ${ }^{33}$ Moreover, we had identified miR-211, among the top miRNAs, by sequencing miRNAs in 19 specimens using whole sections; miR-211 expression was significantly decreased in melanomas compared with melanocytic nevi, which was confirmed in an 
Table 6 Relationship between miR-21-5p expression and prognostic parameters in patients with invasive primary cutaneous melanoma

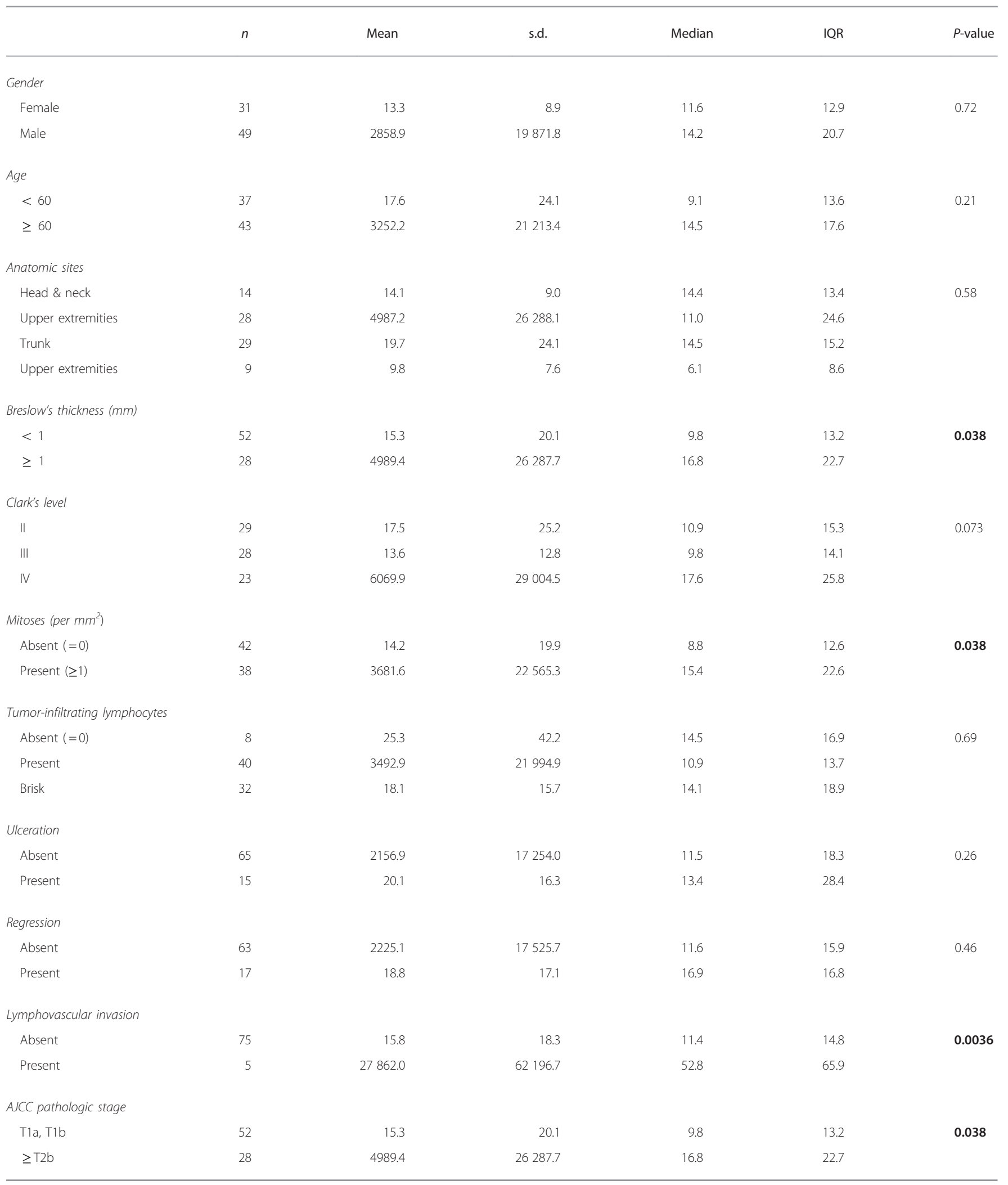

Kruskal-Wallis test was used for the significance of comparison among more than three groups. Bold type signifies statistically significant $P$ values. 

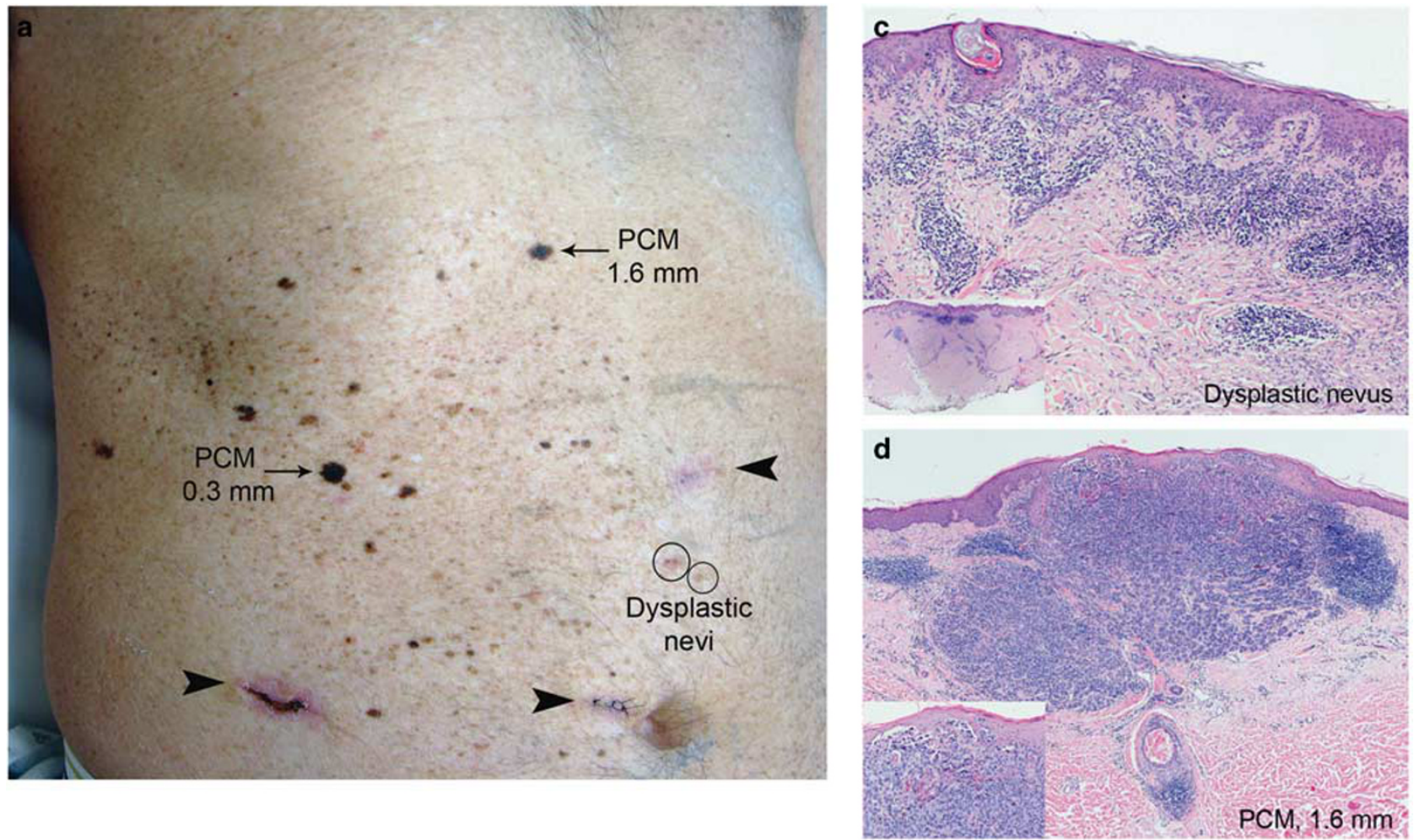

b

\section{Expression of miR-21-5p by qRT-PCR}

miR-21-5p
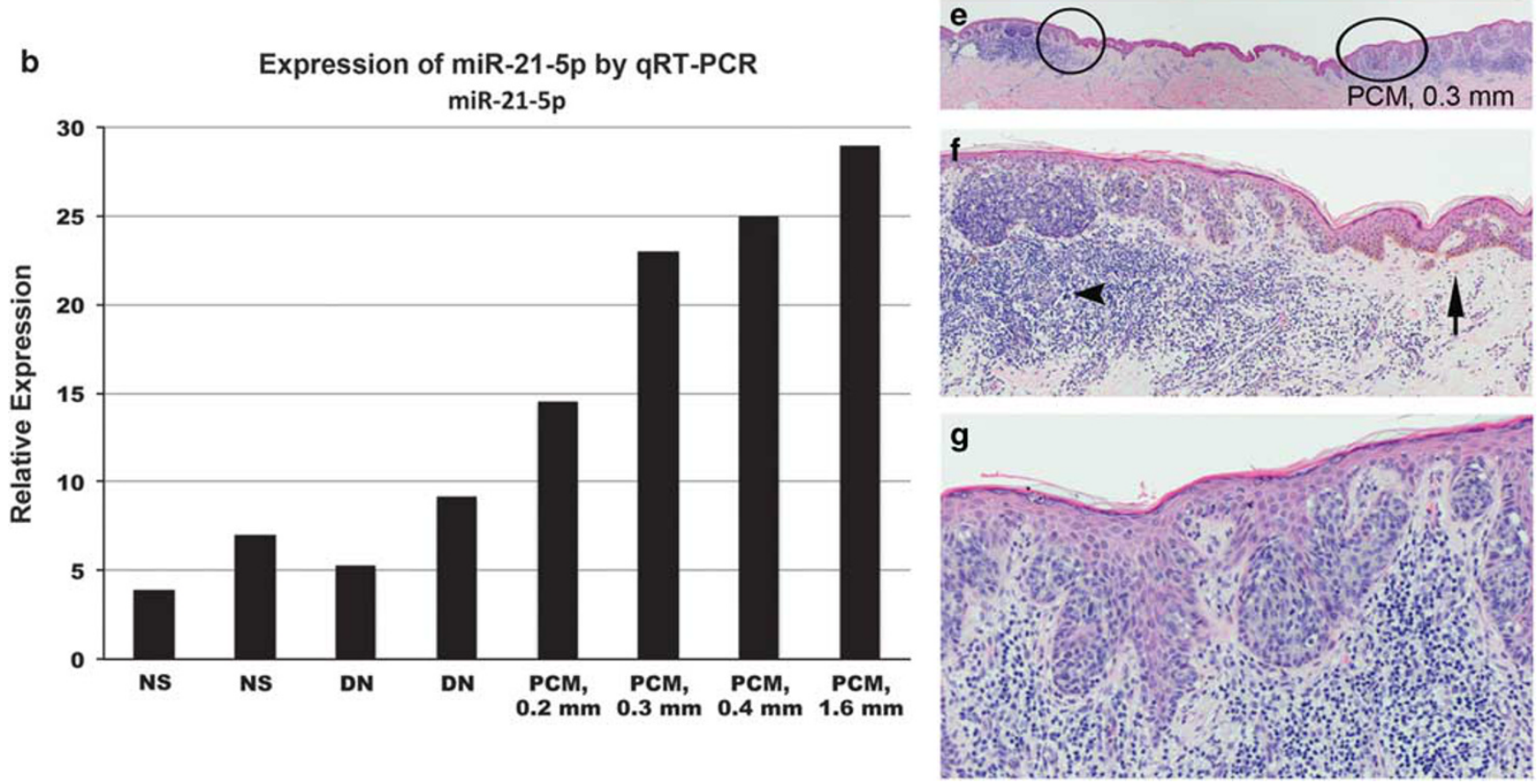

Figure 4 Comparison of increased miR-21-5p expression in multiple lesions and their histopathology from the same individual. (a) The case of a 50-year-old man illustrated developing multiple melanomas and atypical (dysplastic) nevi in a nevus spilus expanding on the right anterior and posterior trunk. Scars (arrowheads) from three prior biopsies were evident. New invasive primary cutaneous melanomas (PCM), with respective invasive depths, and dysplastic nevi (DN) were identified. (b) The expression of miR-21-5p was compared between the DN and previous and current PCMs, comparing with matched normal skin (NS). (c) DN with marked band-like inflammation showed both cytological and architectural atypia. (d) Invasive PCM (1.6 mm) is non-ulcerated with nodular morphology and vertical growth phase. (e) Scanning magnification of two melanomas separated by intervening background skin. (f) Magnified area of circle (in e) showed an invasive component (arrowhead) to a depth of $0.3 \mathrm{~mm}$ in the inflammation and an intervening background skin showed lentigo simplex-like features (arrow). (g) Magnified area of ellipse (in e) showed melanoma in situ component and accompanying dermal inflammation. 
independent validation cohort of 101 specimens by qRTPCR. ${ }^{18}$ Corroborating these findings, miRNA in situ hybridization for miR-211 demonstrated a significant loss of expression in melanomas compared with melanocytic nevi in 109 primary melanocytic tumors. ${ }^{25}$

Using a similar approach, we have defined a spectrum of top 40 miRNAs associated with melanoma invasion (average thickness $2.0 \mathrm{~mm}$ ) into the dermis in clinically annotated specimens in the current study. This specific miRNA signature categorized invasive melanomas into two distinct $0.75 \mathrm{~mm}$ and $2.7 \mathrm{~mm}$ groups. Comparing the miRNA differences between $>4.0 \mathrm{~mm}$ and $2.0 \mathrm{~mm}$ melanomas, in both studies, showed that 6 out of $40(15 \%)$ of miRNAs were shared, whereas the remainder $85 \%$ of the signature was unique to the tumor invasive thickness ${ }^{18}$ and Supplementary Figure S2. This comparison suggests that while the same miRNAs, regulating gene networks, may be involved in the early melanoma invasive process, ultimately unique miRNA changes are needed for disease progression.

Furthermore, the expression of let-7b was significantly decreased in invasive and in situ melanomas compared with normal skin, common, and dysplastic nevi. The decreased let$7 \mathrm{~b}$ expression significantly correlated with invasive depth, Clark's level, ulceration, and AJCC stage. Let-7b belongs to larger let-7 family, whose expression is significantly decreased across a number of human malignancies, including melanoma. An early study demonstrated significant decreased expression of let-7a, let-7b, and let-7d in melanocytic nevi compared with primary melanomas. ${ }^{34}$ Although unclear which histologic subtype or whether invasive or in situ melanoma, investigators experimentally showed that ectopic expression of let-7b in one melanoma cell line (SK-MEL-147) led to decreased expression of cyclins D1, D3, and A, as well as cyclin-dependent kinase. Specific targeting of cyclin D1 $3^{\prime}$ UTR was shown to be the negative regulatory effect of let- $7 \mathrm{~b} .{ }^{34}$ Not surprisingly, decreased expression of these cyclins, by let-7b, reduced cell cycle progression and likely inhibited anchorage-independent growth.

In contrast to let-7b, the measured expressions of miR-21-5p and miR-424-5p were significantly increased in invasive compared to in situ melanomas in an independent validation cohort. The increased expression miR-21-5p was significantly associated with Breslow's depth of tumor invasion. The progressive expression of miR-21-5p could be demonstrated with increasing melanoma thickness in the same individual with multiple melanomas and dysplastic nevi.

miR-21 is commonly deregulated across a variety of solid malignancies, including melanoma; and is established as a potent oncogenic miRNA (oncomiR). One study showed increased expression of miR-21 in melanomas compared with common nevi, which was associated with mitotic activity, ${ }^{35}$ but not invasive depth. In B16 mouse melanoma cells, miR-21 target and functionally inactivate two important tumor suppressor genes, PTEN and PDCD4, where its expression could establish large lung metastases in a tailvein-injection mouse model. ${ }^{36}$ In this model, miR-21 knockdown tumor-bearing mice demonstrated prolonged survival, highlighting the role of this miRNA in tumor growth and metastasis in melanoma. Corroborating our current miRNA sequencing results, a qRT-PCR-based study showed an increased miR-21 expression from dysplastic to primary to metastatic melanomas, which correlated with tumor thickness and advanced clinical stage. ${ }^{37}$ Importantly, in this study, melanoma patients with high miR-21 expression exhibited shorter 5-year disease-free or overall survival compared with those with low expression status. ${ }^{37}$ Furthermore, miR-21 expression can be induced by UV-A and UV-B irradiation in human keratinocytes. ${ }^{38}$ This finding provides a novel mechanism where direct DNA mutations are not necessary for melanomagenesis, instead via epigenetic means; tumor suppressive activity of two normal PTEN alleles can be rendered inactive in a melanoma patient with excessive sun exposure or UV-bed tanning.

Also among the top miRNAs in the current study, defining an invasive behavior, were miR-182-5p and miR-205-5p whose expressions were significantly decreased in thick $(2.7 \mathrm{~mm})$ compared with thin $(0.75 \mathrm{~mm})$ invasive melanomas. The miR-205-5p expression was significantly decreased in primary melanomas and metastases to lymph node compared with nevi. ${ }^{18}$ A large study of primary melanomas $(n=206)$ corroborated miR-205 downregulation and showed a significant association with worse clinical outcome. ${ }^{39}$ In a melanoma cell model, miR-205 overexpression suppressed cell proliferation via targeting E2F1 protein, ${ }^{40}$ a key regulator of G1/S transition phase in the cell cycle. miR-182 is part of a miRNA cluster in 7q31-34 chromosomal locus, frequently amplified in melanoma. In contrast to our current finding, it has been shown that miR-182 is upregulated in melanoma cell lines and specimens, where its overexpression promoted metastatic potential by repressing FOXO3 and microphthalmia-associated transcription factor (MITF) ${ }^{41}$

Overall, emerging results have provided a central role for noncoding RNAs, especially miRNAs in melanoma. Likely, these results will aid in improving melanoma diagnosis in histologically equivocal melanocytic tumors and/or improve melanoma prognostication. Our current study provides additional proof that comprehensive miRNA sequencing strategy combined with qRT-PCR validation of large, independent cohort can provide clinically meaningful results in improving the care for melanoma patients and/or provide novel therapeutic strategies.

Supplementary Information accompanies the paper on the Laboratory Investigation website (http://www.laboratoryinvestigation.org)

\section{ACKNOWLEDGMENTS}

We greatly appreciate Mrs Elizabeth Fleming for technical assistance with sequencing. This work was in part funded by Connecticut Institute for Clinical and Translational Science to SSD. 


\section{DISCLOSURE/CONFLICT OF INTEREST}

The authors declare no conflict of interest.

1. Bartel DP. MicroRNAs: genomics, biogenesis, mechanism, and function. Cell 2004;116:281-297.

2. Calin GA, Sevignani C, Dumitru CD, et al. Human microRNA genes are frequently located at fragile sites and genomic regions involved in cancers. Proc Natl Acad Sci USA 2004;101:2999-3004.

3. Johnson SM, Grosshans H, Shingara J, et al. RAS is regulated by the let7 microRNA family. Cell 2005;120:635-647.

4. Calin GA, Dumitru CD, Shimizu M, et al. Frequent deletions and downregulation of micro-RNA genes miR15 and miR16 at 13q14 in chronic lymphocytic leukemia. Proc Natl Acad Sci USA 2002;99:15524-15529.

5. Tagawa $\mathrm{H}$, Seto M. A microRNA cluster as a target of genomic amplification in malignant lymphoma. Leukemia 2005;19:2013-2016.

6. Hayashita $\mathrm{Y}$, Osada $\mathrm{H}$, Tatematsu $\mathrm{Y}$, et al. A polycistronic microRNA cluster, miR-17-92, is overexpressed in human lung cancers and enhances cell proliferation. Cancer Res 2005;65:9628-9632.

7. Ma Z, Swede H, Cassarino D, et al. Up-regulated Dicer expression in patients with cutaneous melanoma. PLoS One 2011;6:e20494.

8. Hammond SM. Dicing and slicing: the core machinery of the RNA interference pathway. FEBS Lett 2005;579:5822-5829.

9. Mueller DW, Rehli M, Bosserhoff AK. miRNA expression profiling in melanocytes and melanoma cell lines reveals miRNAs associated with formation and progression of malignant melanoma. J Invest Dermatol 2009;129:1740-1751.

10. Caramuta S, Egyhazi S, Rodolfo $\mathrm{M}$, et al. MicroRNA expression profiles associated with mutational status and survival in malignant melanoma. J Invest Dermatol 2010;130:2062-2070.

11. Greenberg E, Hershkovitz L, Itzhaki $O$, et al. Regulation of cancer aggressive features in melanoma cells by microRNAs. PLoS One 2011;6: e18936.

12. Penna $E$, Orso $F$, Cimino $D$, et al. microRNA-214 contributes to melanoma tumour progression through suppression of TFAP2C. EMBO J 2011;30:1990-2007.

13. Chan $E$, Patel $R$, Nallur $S$, et al. MicroRNA signatures differentiate melanoma subtypes. Cell Cycle 2011;10:1845-1852.

14. Segura MF, Belitskaya-Levy I, Rose $A E$, et al. Melanoma MicroRNA signature predicts post-recurrence survival. Clin Cancer Res 2010;16: 1577-1586.

15. Philippidou D, Schmitt M, Moser D, et al. Signatures of microRNAs and selected microRNA target genes in human melanoma. Cancer Res 2010;70:4163-4173.

16. Glud $M$, Rossing $M$, Hother $C$, et al. Downregulation of miR-125b in metastatic cutaneous malignant melanoma. Melanoma Res 2010;20:479-484.

17. Poliseno L, Haimovic A, Segura MF, et al. Histology-specific microRNA alterations in melanoma. J Invest Dermatol 2012;132:1860-1868.

18. Kozubek J, Ma Z, Fleming $E$, et al. In-depth characterization of microRNA transcriptome in melanoma. PLoS One 2013:8:e72699.

19. Mazar J, Qi F, Lee B, et al. MicroRNA 211 functions as a metabolic switch in human melanoma cells. Mol Cell Biol 2016;36:1090-1108.

20. Levy C, Khaled M, lliopoulos D, et al. Intronic miR-211 assumes the tumor suppressive function of its host gene in melanoma. Mol Cell 2010;40:841-849.

21. Mazar J, DeYoung K, Khaitan D, et al. The regulation of miRNA-211 expression and its role in melanoma cell invasiveness. PLoS One 2010;5:e13779.

22. Babapoor $\mathrm{S}$, Fleming $\mathrm{E}, \mathrm{Wu} \mathrm{R}$, et al. A novel miR-451a isomiR, associated with amelanotypic phenotype, acts as a tumor suppressor in melanoma by retarding cell migration and invasion. PLoS One 2014;9:e107502.

23. Duncan LM, Deeds J, Hunter J, et al. Down-regulation of the novel gene melastatin correlates with potential for melanoma metastasis. Cancer Res 1998;58:1515-1520.

24. Duncan LM, Deeds J, Cronin FE, et al. Melastatin expression and prognosis in cutaneous malignant melanoma. J Clin Oncol 2001;19: 568-576.

25. Babapoor S, Horwich M, Wu R, et al. microRNA in situ hybridization for miR-211 detection as an ancillary test in melanoma diagnosis. Mod Pathol 2016;29:461-475.

26. Doeden $\mathrm{K}, \mathrm{Ma} Z$ Z, Narasimhan B, et al. Lymphatic invasion in cutaneous melanoma is associated with sentinel lymph node metastasis. J Cutan Pathol 2009:36:772-780.

27. Ma Z, Lui WO, Fire A, Dadras SS. Profiling and discovery of novel miRNAs from formalin-fixed, paraffin-embedded melanoma and nodal specimens. J Mol Diagn 2009;11:420-429.

28. Tibshirani R, Hastie T, Narasimhan B, et al. Diagnosis of multiple cancer types by shrunken centroids of gene expression. Proc Natl Acad Sci USA 2002;99:6567-6572.

29. Friedlander MR, Chen W, Adamidi C, et al. Discovering microRNAs from deep sequencing data using miRDeep. Nat Biotechnol 2008;26: $407-415$.

30. Witten D, Tibshirani R, Gu SG, et al. Ultra-high throughput sequencingbased small RNA discovery and discrete statistical biomarker analysis in a collection of cervical tumours and matched controls. BMC Biol 2010;8:58

31. Reich M, Liefeld T, Gould J, et al. GenePattern 2.0. Nat Genet 2006;38: 500-501.

32. Falzarano SM, Zhou M, Carver $\mathrm{P}$, et al. Effect of neoadjuvant docetaxel treatment for locally advanced prostate cancer on miRNA expression: a pilot study. J Clin Oncol 2012;30:139.

33. Schou JV, Andersen KK, Jensen BV, et al. Impact of microRNA miR-345 in blood on survival and response in 144 patients with metastatic colorectal cancer treated with third-line cetuximab and irinotecan. $\mathrm{J}$ Clin Oncol 2012;30:451.

34. Schultz J, Lorenz P, Gross $\mathrm{G}$, et al. MicroRNA let-7b targets important cell cycle molecules in malignant melanoma cells and interferes with anchorage-independent growth. Cell Res 2008;18:549-557.

35. Grignol V, Fairchild ET, Zimmerer JM, et al. miR-21 and miR-155 are associated with mitotic activity and lesion depth of borderline melanocytic lesions. Br J Cancer 2011;105:1023-1029.

36. Yang $\mathrm{CH}$, Yue J, Pfeffer SR, et al. MicroRNA miR-21 regulates the metastatic behavior of B16 melanoma cells. J Biol Chem 2011;286: 39172-39178.

37. Jiang $L, L v X$, Li J, et al. The status of microRNA-21 expression and its clinical significance in human cutaneous malignant melanoma. Acta Histochem 2012;114:582-588.

38. Syed DN, Khan Ml, Shabbir M, et al. MicroRNAs in skin response to UV radiation. Curr Drug Targets 2013;14:1128-1134.

39. Hanna JA, Hahn L, Agarwal S, et al. In situ measurement of miR-205 in malignant melanoma tissue supports its role as a tumor suppressor microRNA. Lab Invest 2012;92:1390-1397.

40. Dar AA, Majid S, de Semir D, et al. miRNA-205 suppresses melanoma cell proliferation and induces senescence via regulation of E2F1 protein. J Biol Chem 2011;286:16606-16614.

41. Segura MF, Hanniford D, Menendez S, et al. Aberrant miR-182 expression promotes melanoma metastasis by repressing $\mathrm{FOXO} 3$ and microphthalmia-associated transcription factor. Proc Natl Acad Sci USA 2009;106:1814-1819. 